Publ. Mat. 47 (2003), 441-450

\title{
VOLUMES TRANSVERSES AUX FEUILLETAGES DÉFINISSABLES DANS DES STRUCTURES O-MINIMALES
}

\author{
F. Chazal And J.-M. LiON
}

\begin{abstract}
Let $\mathcal{F}_{\lambda}$ be a family of codimension $p$ foliations defined on a family $M_{\lambda}$ of manifolds and let $X_{\lambda}$ be a family of compact subsets of $M_{\lambda}$. Suppose that $\mathcal{F}_{\lambda}, M_{\lambda}$ and $X_{\lambda}$ are definable in an o-minimal structure and that all leaves of $\mathcal{F}_{\lambda}$ are closed. Given a definable family $\Omega_{\lambda}$ of differential $p$-forms satisfaying $i_{Z} \Omega_{\lambda}=0$ for any vector field $Z$ tangent to $\mathcal{F}_{\lambda}$, we prove that there exists a constant $A>0$ such that the integral of $\left|\Omega_{\lambda}\right|$ on any transversal of $\mathcal{F}_{\lambda}$ intersecting each leaf in at most one point is bounded by $A$. We apply this result to prove that $p$-volumes of transverse sections of $\mathcal{F}_{\lambda}$ are uniformly bounded.
\end{abstract}

\section{Introduction}

L'objet de ce travail est d'étudier le volume des sections transverses aux feuilletages définissables dans une structure o-minimale et dont toutes les feuilles sont fermées. Nous généralisons des résultats de D'Acunto et Kurdyka relatifs aux fonctions [DK] et de Kurdyka relatifs aux applications [K1], [K2]. L'exemple suivant illustre le type de résultats que nous obtenons. Considérons une 1-forme $\omega$ de $\mathbf{R}^{n}$ à coefficients semi-algébriques et continuement dérivables. Supposons que $\omega$ est intégrable $(\omega \wedge d \omega=0)$. Soit $X$ un ouvert semi-algébrique borné de $\mathbf{R}^{n}$ sur lequel $\omega$ est non singulière. L'équation de Pfaff $\omega=0$ définit un feuilletage $\mathcal{F}$ de codimension un sur $X$. Supposons que les feuilles de $\mathcal{F}$ sont fermées. Nous montrons l'existence d'une courbe semi-algébrique $\gamma$ contenue dans $\bar{X}$ telle que si $C$ est une courbe de $X$ vérifiant

$$
\int_{C}|\omega|>\int_{\gamma}|\omega|
$$

2000 Mathematics Subject Classification. 53C12, 49Q15, 03C64, 32B20.

Mots-clés. Feuilletages réels, structures o-minimales, intégration de formes différentielles.

Partiellement financé par RAAG HPRN-CT-00271. 
alors $C$ rencontre une feuille de $\mathcal{F}$ en au moins deux points. En particulier, la longueur de toute courbe orthogonale (pour la métrique euclidienne) à $\mathcal{F}$ coupant au plus une fois chaque feuille est majorée par la longueur de $\gamma$. Nous montrons que ce résultat reste vrai pour un feuilletage de codimension quelconque définissable dans une structure o-minimale. Il reste également vrai si dans l'inégalité précédente $\omega$ est remplacée par une forme $\Omega$ annulée par tout champ de vecteurs tangent à $\mathcal{F}$.

Définition. Soit $\mathcal{F}$ un feuilletage de codimension $p$ d'une sous-variété $M$ de classe $C^{2}$ de $\mathbf{R}^{n}$. On dit qu'une $p$-forme $\Omega$ sur $M$ est annulée par tout champ tangent à $\mathcal{F}$ si $i_{Z} \Omega=0$ pour tout champ de vecteurs $Z$ tangent à $\mathcal{F}$.

Avant d'énoncer notre théorème, donnons brièvement la définition et les propriétés essentielles des structures o-minimales. Pour plus de détails nous renvoyons le lecteur à $[\mathbf{D r M}]$ ou $[\mathbf{D r 2}]$ par exemple. Une structure o-minimale $\mathcal{A}$ est une famille de sous-algèbres de Boole $\mathcal{A}_{n} \subset$ $\mathcal{P}\left(\mathbf{R}^{n}\right)$ qui contient les sous-ensembles semi-algébriques, qui est stable par produit et projection canonique et dont les éléments ont un nombre fini de composantes connexes. D'après un théorème de Tarski [Ta] la famille des semi-algébriques des espaces $\mathbf{R}^{n}$ est une structure o-minimale. Gabrièlov [Ga] montre que les sous-analytiques globaux des espaces $\mathbf{R}^{n}$ constituent une seconde structure o-minimale. Un sous-ensemble de $\mathbf{R}^{n}$ est dit $\mathcal{A}$-définissable (ou définissable dans $\mathcal{A}$ ) si c'est un élément de $\mathcal{A}_{n}$. Une fonction de graphe $\mathcal{A}$-définissable est dite $\mathcal{A}$-définissable (ou définissable dans $\mathcal{A}$ ). On définit de la même façon les applications et les formes $\mathcal{A}$-définissables: chaque fonction coordonnée de l'application ou chaque coefficient de la forme sont des fonctions $\mathcal{A}$-définissables.

Dans la suite $\mathcal{A}$ désigne une structure o-minimale fixée. Tout sousensemble $\mathcal{A}$-définissable $C$ est $\mathcal{A}$-stratifiable (voir $[\mathbf{D r M}]$ ou $[\mathbf{D r 2}]$ ). Sa dimension est donc bien définie. Ceci implique que si $\Omega$ est une $p$-forme $\mathcal{A}$-définissable, si $C$ est de dimension au plus $p$ et si $C_{0}$ est un sousensemble mesurable de $C$ alors l'intégrale $\int_{C_{0}}|\Omega|$ est bien définie. Si $X_{\lambda}$ est une famille de sous-ensembles $\mathcal{A}$-définissables de $\mathbf{R}^{n}$ qui dépendent de façon $\mathcal{A}$-définissable du paramètre $\lambda$ alors il existe un entier qui majore le nombre de composantes connexes de chaque $X_{\lambda}$ (voir $[\mathbf{D r M}]$ ou [Dr2]). Si de plus les $X_{\lambda}$ sont de dimension au plus $p$ et sont inclus dans un même compact alors il existe une constante qui majore le volume $p$-dimensionnel de chacun d'eux. Ceci résulte de la propriété de finitude uniforme précédente combinée à la formule de Cauchy-Crofton (voir $[\mathbf{F e}]$ ou $[\mathbf{C Y}]$ ). 
Soit $M$ une sous-variété $\mathcal{A}$-définissable de classe $C^{2}$ de $\mathbf{R}^{n}$.

Définition. Un feuilletage $\mathcal{F}$ de codimension $p$ sur $M$ est dit $\mathcal{A}$-définissable s'il existe un recouvrement fini de $M$ par des ouverts $\mathcal{A}$-définissables tels qu'en restriction à chacun d'eux $\mathcal{F}$ soit défini par un système de Pfaff $\left(\omega_{1}, \ldots, \omega_{p}\right) \mathcal{A}$-définissable de classe $C^{1}$ :

$$
\omega_{1} \wedge \cdots \wedge \omega_{p} \neq 0 \quad \text { et } \quad d \omega_{i} \wedge\left(\bigwedge_{j \neq i} \omega_{j}\right)=0 .
$$

Depuis $[\mathbf{D r} \mathbf{1}],[\mathbf{K h}]$ et $[\mathbf{M R}]$ les feuilles de certains feuilletages $\mathcal{A}$ définissables de codimension 1 et transversalement orientés (appelés feuilletages de Rolle) ont été largement étudiées. La structure topologique de l'espace des feuilles est un arbre fini $[\mathbf{C h}]$. Ces feuilles engendrent une structure o-minimale ([Sp], voir aussi $[\mathbf{W i}],[\mathbf{L R}],[\mathbf{K M}])$. Le résultat que nous prouvons est relatif à la structure métrique de l'espace des feuilles des feuilletages $\mathcal{A}$-définissables de codimension quelconque et dont les feuilles sont fermées.

Théorème 1. Soit $\mathcal{F}$ un feuilletage de codimension $p$ sur une sousvariété $M$ de classe $C^{2}$ de $\mathbf{R}^{n}$, soit $X$ un sous-ensemble compact de $M$ et soit $\Omega$ une p-forme définie sur $M$, annulée par tout champ tangent à $\mathcal{F}$. On suppose que $M, X, \mathcal{F}$ et $\Omega$ sont $\mathcal{A}$-définissables et que les feuilles de $\mathcal{F}$ sont fermées. Il existe $\Gamma \subset X$ un sous-ensemble définissable de dimension $p$ vérifiant la propriété suivante: si $C$ est une sous-variété de dimension $p$ contenue dans $X$ telle que

$$
\int_{C}|\Omega|>\int_{\Gamma}|\Omega|
$$

alors $C$ rencontre une feuille de $\mathcal{F}$ en au moins deux points.

Théorème 2 (version à paramètres). Dans l'énoncé précédent si $M=M_{\lambda}, X=X_{\lambda}, \mathcal{F}=\mathcal{F}_{\lambda}$ et $\Omega=\Omega_{\lambda}$ dépendent de façon $\mathcal{A}$-définissable d'un paramètre $\lambda \in \mathbf{R}^{d}$ alors $\Gamma=\Gamma_{\lambda}$ dépend de façon définissable de $\lambda$.

Le Théorème 1 est un corollaire immédiat du Théorème 2. Nous ne prouverons donc que ce dernier. Notre preuve consiste à remarquer que $\Omega$ définit une $p$-forme sur le fibré normal $T X / T \mathcal{F}$ à $\mathcal{F}$ et à établir une relation entre l'application d'holonomie de $\mathcal{F}$ au voisinage d'une feuille sans holonomie et l'intégrale de $\Omega$ sur une section transverse (formule (2) ci-dessous). Cette approche est différente de celle adoptée dans [DK], $[\mathbf{K 1}],[\mathbf{K 2}]$ et nous permet d'obtenir un résultat ne faisant intervenir aucune hypothèse de nature métrique sur la variété $M$. 
Ce travail doit beaucoup à des conversations avec D. D'Acunto, K. Kurdyka et R. Moussu que nous remercions.

\section{Application aux volumes de sections transverses et théorème de D'Acunto-Kurdyka}

Dans cette section nous montrons comment notre Théorème 2 permet de retrouver les résultats de $[\mathbf{D K}],[\mathbf{K 1}],[\mathbf{K 2}]$ qui généralisent un célèbre théorème de Łojasiewicz sur les longueurs des trajectoires de gradients analytiques $[\mathbf{E o}]$ et plus généralement comment il permet de majorer les volumes des sections transverses à des familles $\mathcal{A}$-définissables de feuilletages $\mathcal{A}$-définissables.

Théorème $([\mathbf{D K}],[\mathbf{K} \mathbf{1}],[\mathbf{K 2}])$. Soit $d \in \mathbf{N}$ et soit $K \in] 0,1]$. Il existe une constante $A>0$ vérifiant la propriété suivante. Soit $P: \mathbf{R}^{n} \rightarrow$ $\mathbf{R}^{p}$ une application polynomiale de degré $d$ et soit $C$ une sous-variété de dimension $p$ contenue dans la boule unité $B$ de $\mathbf{R}^{n}$. Les niveaux de $P$ définissent un feuilletage singulier $\mathcal{F}$ sur $B$. Supposons que $C$ est transverse à $\mathcal{F}$ et que pour tout $x \in C$ la restriction $\grave{a} T_{x} C$ de la projection orthogonale de $\mathbf{R}^{n}$ (muni de la structure euclidienne usuelle) sur l'espace orthogonal à $T_{x} \mathcal{F}$ est de déterminant supérieur à $K$. Alors ou bien le volume p-dimensionnel de $C$ est inférieur ou égal à $A$, ou bien $C$ coupe deux fois une feuille de $\mathcal{F}$.

Preuve: En appliquant le Théorème 2 à la famille à paramètre $(P, \varepsilon)=$ $\left(P_{1}, \ldots, P_{p}, \varepsilon\right) \in \mathbf{R}\left[X_{1}, \ldots, X_{n}\right]^{p} \times \mathbf{R}^{+}$:

$$
\begin{aligned}
& M_{P}=\mathbf{R}^{n} \backslash\{d P=0\}, \\
& \Omega_{P}=\frac{d P_{1} \wedge \cdots \wedge d P_{p}}{\left\|d P_{1} \wedge \cdots \wedge d P_{p}\right\|}
\end{aligned}
$$

et

$$
X_{P, \varepsilon}=\{x \in B \mid d(x,\{d P=0\}) \geq \varepsilon\},
$$

nous obtenons une famille semi-algébrique de sous-ensembles semi-algébriques $\Gamma_{P, \varepsilon}$ de dimension au plus $p$ contenus dans $B$. Soit $C$ comme dans l'énoncé ci-dessus. Supposons que $C$ coupe au plus une fois chaque niveau de $f$. D'après le Théorème 2 on a

$$
\int_{\Gamma_{P, \varepsilon}}\left|\Omega_{P}\right| \geq \int_{C}\left|\Omega_{P}\right| .
$$


Or il existe une constante $A_{0}$ qui majore uniformément les volumes $p$-dimensionnels de ces sous-ensembles et par construction de $\Omega_{P}$, le volume $p$-dimensionnel de $\Gamma_{P, \varepsilon}$ majore l'intégrale $\int_{\Gamma_{P, \varepsilon}}\left|\Omega_{P}\right|$. De plus, puisque pour tout $x \in C$ la restriction à $T_{x} C$ de la projection orthogonale de $\mathbf{R}^{n}$ sur l'espace orthogonal à $T_{x} \mathcal{F}$ est de déterminant supérieur à $K$, l'intégrale $\int_{C}\left|\Omega_{P}\right|$ est minorée par $K$ fois le volume $p$-dimensionnel de $C$. Par conséquent le volume $p$-dimensionnel de $C$ est majoré par $A=A_{0} / K$.

Remarquons que le résultat précédent reste valable pour une famille $\mathcal{A}$-définissable d'applications $\mathcal{A}$-définissables.

Si dans l'énoncé du Théorème 2 , on suppose de plus que la famille $M_{\lambda}$ est munie d'une famille $\mathcal{A}$-définissable de métriques riemmaniennes $\mathcal{A}$-définissables, nous obtenons le corollaire suivant qui généralise aux feuilletages le résultat de [DK], [K1], [K2] énoncé précédemment.

Corollaire. Soit $K \in] 0 ; 1\left[\right.$, soit $\mathcal{F}_{\lambda}$ une famille de feuilletages de codimension $p$ définie sur une famille $M_{\lambda}$ de sous-variétés de classe $C^{2}$ de $\mathbf{R}^{n}$ munies d'une métrique riemmanienne $g_{\lambda}$ et soit $X_{\lambda}$ une famille de sous-ensembles compacts de $M_{\lambda}$. On suppose que $M_{\lambda}, X_{\lambda}, \mathcal{F}_{\lambda}$ et $g_{\lambda}$ sont $\mathcal{A}$-définissables et dépendent de manière $\mathcal{A}$-définissable du paramètre $\lambda$ et que les feuilles de $\mathcal{F}_{\lambda}$ sont fermées. Il existe une constante $A>0$ vérifiant la propriété suivante. Soit $C_{\lambda}$ une sous-variété de $X_{\lambda}$ de dimension $p$ transverse à $\mathcal{F}$ telle que pour tout $x \in C_{\lambda}$ la restriction à $T_{x} C_{\lambda}$ de la projection orthogonale de $T_{x} M_{\lambda}$ sur l'espace orthogonal à $T_{x} \mathcal{F}_{\lambda}$ est de déterminant supérieur à $K$. Alors, ou bien le volume $p$-dimensionnel de $C_{\lambda}$ est inférieur ou égal à $A$, ou bien $C_{\lambda}$ coupe deux fois une feuille de $\mathcal{F}_{\lambda}$.

Preuve: C'est une adaptation immédiate de la preuve précédente en prenant pour $\Omega=\Omega_{\lambda}$ la $p$-forme volume induite par la métrique de $M_{\lambda}$ sur le fibré orthogonal à $T \mathcal{F}_{\lambda}$.

Remarquons enfin que dans le cas de feuilletages algébriques la constante $A$ du corollaire ne dépend que du degré des polynômes définissant $\mathcal{F}_{\lambda}$ : si $\mathcal{F}_{\lambda}$ est une famille de feuilletages de codimension $p$ de $\mathbf{R}^{n}$ définie par un système de Pfaff dont les coefficients sont des polynômes de degré au plus $d \in \mathbf{N}$ et si les sous-ensembles $M_{\lambda}$ sont contenus dans une boule de rayon $r>0$ fixé, alors la constante $A$ peut être choisie de la forme $A=r A(n, d)$ où $A(n, d)$ est une constante ne dépendant que de $n$ et de $d$. En effet, dans ce cas les sous-ensembles $\Gamma_{\lambda}$ sont semi-algébriques (voir la définition de $\Gamma_{\lambda}$ dans la section suivante) et leur degré ne dépend que du degré du système de Pfaff définissant $\mathcal{F}_{\lambda}$. 


\section{Démonstration du Théorème 2}

D'après les résultats sur les stratifications adaptées à un système de Pfaff [MR] (voir aussi $[\mathbf{L R}],[\mathbf{S p}]$ et $[\mathbf{L S}]$ ) il existe une famille finie $X_{\lambda}^{i}$, $i \in\{1, \ldots, r\}$, de sous-variétés de classe $C^{2} \mathcal{A}$-définissables, un entier $s \leq r$ et une famille finie de feuilletages $\mathcal{A}$-définissables $\mathcal{F}_{\lambda}^{i}$ tels que pour tout $\lambda$ :

1. $X_{\lambda}^{1} \cup \cdots \cup X_{\lambda}^{r}$ est une partition finie de $X_{\lambda}$.

2. $\mathcal{F}_{\lambda}^{i}$ est un feuilletage sur $X_{\lambda}^{i}$ de codimension au plus $p$ et il est de codimension $p$ si et seulement si $i \leq s$.

3. si $V$ est une feuille de $\mathcal{F}_{\lambda}$ alors $V \cap X_{\lambda}^{i}$ est une union de feuilles de $\mathcal{F}_{\lambda}^{i}$.

Soit $i \in\{1, \ldots, r\}$ et $\lambda \in \mathbf{R}^{d}$. Si $i>s$ on pose $Y_{\lambda}^{i}=\emptyset$. Si $i \leq s$ on note $Y_{\lambda}^{i}$ le sous-ensemble de $X_{\lambda}^{i}$ suivant: un point $y$ de $X_{\lambda}^{i}$ est dans $Y_{\lambda}^{i}$ si et seulement si pour tout champ de vecteurs $Z$ sur $X_{\lambda}^{i}$ tangent à $\mathcal{F}_{\lambda}^{i}$ la dérivée de Lie $L_{Z}\left(\Omega_{\lambda}^{i}\right)$ de la $p$-forme $\Omega_{\lambda}^{i}$ induite par $\Omega_{\lambda}$ sur $X_{\lambda}^{i}$ est nulle en $y$. Montrons que $Y_{\lambda}^{i}$ est un ensemble $\mathcal{A}$-définissable. Puisque $\Omega_{\lambda}$ est annulée par tout champ tangent à $\mathcal{F}_{\lambda}$, la forme $\Omega_{\lambda}^{i}$ est annulée par tout champ tangent à $\mathcal{F}_{\lambda}^{i}$ et $L_{Z}\left(\Omega_{\lambda}^{i}\right)(y)$ ne dépend que de la valeur de $Z$ en $y$. En effet, si $Z_{1}$ et $Z_{2}$ sont deux champs de vecteurs tangents à $\mathcal{F}_{\lambda}^{i}$ et si $g$ est une fonction $C^{1}$ alors

$$
L_{g Z_{1}+Z_{2}}\left(\Omega_{\lambda}^{i}\right)=g L_{Z_{1}}\left(\Omega_{\lambda}^{i}\right)+L_{Z_{2}}\left(\Omega_{\lambda}^{i}\right) .
$$

Or, d'après $[\mathbf{L S}]$, l'application $\Pi_{\lambda}^{i}$ qui à $x \in X_{\lambda}^{i}$ associe la projection orthogonale sur le plan tangent $T_{x}\left(\mathcal{F}_{\lambda}^{i}\right)$ au point $x$ de la feuille de $\mathcal{F}_{\lambda}^{i}$ passant par $x$ est de classe $C^{2}$ et $\mathcal{A}$-définissable. Ainsi les champs de vecteurs $Z_{\lambda, k}^{i}=\Pi_{\lambda}^{i}\left(\frac{\partial}{\partial x_{k}}\right), k \in\{1, \ldots, n\}$ sont de classe $C^{2}, \mathcal{A}$-définissables et engendrent en tout point $x \in X_{\lambda}^{i}$ le plan tangent $T_{x}\left(\mathcal{F}_{\lambda}^{i}\right)$. Par conséquent, $Y_{\lambda}^{i}=X_{\lambda}^{i} \cap\left(\cap_{k=1}^{n}\left\{L_{Z_{\lambda, k}^{i}}\left(\Omega_{\lambda}^{i}\right)=0\right\}\right)$. Il est $\mathcal{A}$-définissable.

D'après le lemme de section de Gabrièlov $[\mathbf{G a}]$ adapté aux feuilletages analytiques par $[\mathbf{M R}]$ (voir aussi $[\mathbf{L R}]$ ) et aux feuilletages $\mathcal{A}$-définissables par $[\mathbf{S p}]$ (voir aussi $[\mathbf{L S}]$ ), il existe une famille $\mathcal{A}$-définissable $\tilde{X}_{\lambda}^{i} \subset X_{\lambda}^{i}$, $i \in\{s+1, \ldots, t\}$, de sous-ensembles $\mathcal{A}$-définissables, tels que $\operatorname{dim} \tilde{X}_{\lambda}^{i}<p$ et tels que si $V$ est une feuille de $\mathcal{F}_{\lambda}^{i}$ alors $V \cap \tilde{X}_{\lambda}^{i}$ est discret et rencontre toutes les composantes connexes de $V \cap X_{\lambda}^{i}$. Il existe aussi une famille $\mathcal{A}$-définissable $\Gamma_{\lambda}^{i} \subset Y_{\lambda}^{i}, i \in\{1, \ldots, s\}$, de sous-ensembles $\mathcal{A}$-définissables de dimension $p$, tels que si $V$ est une feuille de $\mathcal{F}_{\lambda}^{i}$ alors $V \cap \Gamma_{\lambda}^{i}$ est discret et rencontre toutes les composantes connexes de $V \cap Y_{\lambda}^{i}$.

En utilisant encore les résultats de stratification adaptée à un feuilletage, on décompose les $\Gamma_{\lambda}^{i}, i \in\{1, \ldots, s\}$, de la façon suivante: 
1. $\Gamma_{\lambda}^{i}=\Gamma_{\lambda}^{\prime i} \cup \Gamma_{\lambda}^{\prime \prime} i$ avec $\Gamma_{\lambda}^{\prime i}$ sous-variété $\mathcal{A}$-définissable, de classe $C^{2}$, de dimension $p$, transverse à $\mathcal{F}_{\lambda}$ et $\Gamma_{\lambda}^{\prime \prime} i$ sous-ensemble $\mathcal{A}$-définissable, de dimension strictement inférieure à $p$.

2. $\Gamma_{\lambda}=\Gamma_{\lambda}^{\prime 1} \cup \cdots \cup \Gamma_{\lambda}^{\prime} s$ est une sous-variété de dimension $p$.

On pose $\tilde{X}_{\lambda}=\left(\cup_{i \leq s} \Gamma_{\lambda}^{\prime \prime} i\right) \cup\left(\cup_{i>s} \tilde{X}_{\lambda}^{i}\right)$. C'est un sous-ensemble $\mathcal{A}$-définissable de dimension strictement inférieure à $p$. Nous allons montrer que l'ensemble $\Gamma_{\lambda}$ décrit ci-dessus satisfait à la conclusion du Théorème 2.

Soit $C$ une sous-variété de dimension $p$ contenue dans $X_{\lambda}$ qui coupe chaque feuille de $\mathcal{F}_{\lambda}$ en au plus un point. Nous allons montrer que $\int_{C}\left|\Omega_{\lambda}\right| \leq \int_{\Gamma_{\lambda}}\left|\Omega_{\lambda}\right|$.

La forme $\Omega_{\lambda}$ étant annulée par tout champ tangent à $\mathcal{F}_{\lambda}$, on a

$$
\int_{C}\left|\Omega_{\lambda}\right|=\int_{C^{\prime}}\left|\Omega_{\lambda}\right|
$$

où $C^{\prime}$ est l'ensemble (ouvert dans $C$ ) des points où $C$ est transverse à $\mathcal{F}_{\lambda}$. Nous pouvons donc supposer que $C$ est transverse à $\mathcal{F}_{\lambda}$. Soit $C^{\prime \prime}$ l'ensemble des points de $C$ où $\Omega_{\lambda}$ est nulle. C'est un fermé de $C$ et $\int_{C^{\prime \prime}}\left|\Omega_{\lambda}\right|=0$. Nous pouvons donc supposer que $\Omega_{\lambda}$ ne s'annule en aucun point de $C$. Soit $C^{\prime \prime \prime}$ l'ensemble mesurable des points de $c \in C$ tels que $V$, la feuille de $\mathcal{F}_{\lambda}$ passant par $c$, rencontre $\tilde{X}_{\lambda}$. Puisque $\operatorname{dim} \tilde{X}_{\lambda}<p$ et que $C$ est de dimension $p$ et transverse à $\mathcal{F}_{\lambda}$, l'ensemble $C^{\prime \prime \prime}$ est de mesure $p$-dimensionnelle nulle et $\int_{C^{\prime \prime \prime}}\left|\Omega_{\lambda}\right|=0$. Par construction des $\tilde{X}_{\lambda}^{i}$, un point $c$ est dans $C^{\prime \prime \prime}$ dès que la feuille $V$ qui passe par ce point rencontre l'un des $X_{\lambda}^{i}$ (et donc l'un des $\tilde{X}_{\lambda}^{i}$ ) si $i>s$. On pose $C_{0}=C \backslash C^{\prime \prime \prime}$. C'est un sous-ensemble mesurable de $C$ et $\int_{C}\left|\Omega_{\lambda}\right|=\int_{C_{0}}\left|\Omega_{\lambda}\right|$. Il suffit donc de montrer $\int_{C_{0}}\left|\Omega_{\lambda}\right| \leq \int_{\Gamma_{\lambda}}\left|\Omega_{\lambda}\right|$.

Soit $c$ un point de $M, S_{c}$ une section transverse à $\mathcal{F}_{\lambda}$ en $c$ coupant au plus une fois chaque feuille et soit $x$ un point appartenant à la feuille $V$ passant par $c$. On suppose $\Omega_{\lambda}(c) \neq 0$. La restriction de $\mathcal{F}_{\lambda}$ au saturé de $S_{c}$ par $\mathcal{F}_{\lambda}$ est un feuilletage sans holonomie (voir [CL] par exemple). Si $S$ est une section du feuilletage en $x$, la différentielle de l'application d'holonomie entre $S_{c}$ et $S$ induit un isomorphisme linéaire entre les fibres $\left(T X_{\lambda} / T \mathcal{F}_{\lambda}\right)_{c}$ et $\left(T X_{\lambda} / T \mathcal{F}_{\lambda}\right)_{x}$ du fibré normal à $\mathcal{F}_{\lambda}$. Cette application est indépendante du choix des sections $S_{c}$ et $S$. D'autre part, $\Omega_{\lambda}$ qui est annulée par tout champ de vecteurs tangent à $\mathcal{F}_{\lambda}$ définit une $p$-forme linéaire sur le fibré normal $T X_{\lambda} / T \mathcal{F}_{\lambda}$. Soit $v=\left(v_{1}, \ldots, v_{p}\right)$ un $p$-uplet de vecteurs formant une base de $\left(T X_{\lambda} / T \mathcal{F}_{\lambda}\right)_{c}$ et soit $w$ l'image de $v$ par la différentielle de l'application d'holonomie entre $S_{c}$ et $S$. Puisque $\Omega_{\lambda}(c) \neq 0$, on a $\Omega_{\lambda}(c) \cdot(v) \neq 0$. Nous définissons une application $\Theta_{c}$ 
sur $V$ par

$$
\Theta_{c}(x)=\frac{\Omega_{\lambda}(x) \cdot(w)}{\Omega_{\lambda}(c) \cdot(v)} .
$$

C'est une application indépendante du choix de $v$. De plus $\Theta_{c}$ est différentiable et si $Z$ est un champ de vecteurs tangent à $V$, on a

$$
Z\left(\Theta_{c}\right)(x)=\frac{L_{Z} \Omega_{\lambda}(x) \cdot(w)}{\Omega_{\lambda}(c) \cdot(v)} .
$$

Si $c^{\prime}$ est un autre point de $V$ tel que $\Omega_{\lambda}\left(c^{\prime}\right) \neq 0$, on a le cocycle multiplicatif $\Theta_{c^{\prime}}(x)=\Theta_{c^{\prime}}(c) \Theta_{c}(x)$. Soit $S_{c^{\prime}}$ une section transverse de $\mathcal{F}_{\lambda}$ passant par $c^{\prime}$. En restreignant éventuellement $S_{c}$ et $S_{c^{\prime}}$, il existe un difféomorphisme d'holonomie $h$ entre $S_{c}$ et $S_{c^{\prime}}$. On a donc la formule $d e$ changement de variable

$$
\int_{S_{c^{\prime}}}\left|\Omega_{\lambda}\right|=\int_{S_{c}}\left|\Omega_{\lambda}(x)\right|\left|\Theta_{x}(h(x))\right| .
$$

On en déduit le lemme suivant qui est dans l'esprit de [Go, Théorème 1.5 et Proposition 1.6, p. 140].

Lemme. Soit $C_{1}$ une sous-variété de dimension $p$ contenue dans $X_{\lambda}$, transverse à $\mathcal{F}_{\lambda}$. Supposons que pour tout $x \in C_{0}$, il existe un point $x^{\prime} \in C_{1}$ appartenant à la même feuille que $x$ et telle que $\left|\Theta_{x}\left(x^{\prime}\right)\right| \geq 1$. Alors

$$
\int_{C_{0}}\left|\Omega_{\lambda}\right| \leq \int_{C_{1}}\left|\Omega_{\lambda}\right| .
$$

De plus, si $c$ est un point d'une strate $X_{\lambda}^{i}$ de $X_{\lambda}$ sur laquelle $\mathcal{F}_{\lambda}^{i}$ est de codimension $p$ on définit de même une application $\Theta_{c}^{i}$ sur la feuille $V^{i}$ de $\mathcal{F}_{\lambda}^{i}$ qui passe par $c$. Le fibré normal à $\mathcal{F}_{\lambda}^{i}$ coïncide avec la restriction à $X_{\lambda}^{i}$ du fibré normal à $\mathcal{F}_{\lambda}$. L'application $\Theta_{c}^{i}$ est donc la restriction de $\Theta_{c}$ à $V^{i}$.

Nous pouvons enfin montrer que $\int_{C_{0}}\left|\Omega_{\lambda}\right| \leq \int_{\Gamma_{\lambda}}\left|\Omega_{\lambda}\right|$ et ainsi terminer la preuve du Théorème 2. Soit $c \in C_{0}$. La restriction de $\left|\Theta_{c}\right|$ au compact $V \cap X_{\lambda}$ atteint son maximum en un point $m$. Soit $X_{\lambda}^{i}$ la strate de $X_{\lambda}$ qui contient $m$. Puisque $c \in C_{0}$, alors $i \leq s,\left|\Omega_{\lambda}\right|$ est non nul en $c$ et en $m$ et $\mathcal{F}_{\lambda}^{i}$ est de codimension $p$. Le point $m$ est aussi un maximum de $\left|\Theta_{m}^{i}\right|$. Il est donc contenu dans $Y_{\lambda}^{i}$. Soit $W$ la composante connexe de $V \cap Y_{\lambda}^{i}$ qui contient $m$. D'après les résultats de stratification adaptée à un feuilletage $[\mathbf{M R}],[\mathbf{S p}],[\mathbf{L R}],[\mathbf{L S}], W$ se décompose en une union dénombrable de sous-variétés sur lesquelles $\left|\Theta_{m}^{i}\right|$ et $\left|\Theta_{c}\right|$ sont constantes. Ceci implique que $\left|\Theta_{m}^{i}\right|$ et $\left|\Theta_{c}\right|$ restreintes à $W$ sont constantes. Par conséquent, $\left|\Theta_{c}\right|$ atteint son maximum en un point $m^{\prime} \in V \cap \Gamma_{\lambda}^{i}$. Puisque $c \in C_{0}$, c'est un point de $\Gamma_{\lambda}^{\prime i}$ et donc de $\Gamma_{\lambda}$. Puisque $\Theta_{c}(c)=1$, 
on a $\left|\Theta_{c}\left(m^{\prime}\right)\right| \geq 1$. Le lemme appliqué à $C_{1}=\Gamma_{\lambda}$ achève la preuve du Théorème 2 .

\section{Bibliographie}

[CL] C. Camacho et A. Lins Neto, "Geometric theory of foliations", Birkhäuser Boston, Inc., Boston, MA, 1985.

[Ch] F. Chazal, Sur les feuilletages algébriques de Rolle, Comment. Math. Helv. 72(3) (1997), 411-425.

[CY] G. Comte et Y. Yomdin, Tame geometry with application in smooth analysis, Preprint, Université de Nice (2002).

[DK] D. D'Acunto et K. Kurdyka, Gradient of a definable family of functions: uniform bound on the length of the trajectories, Preprint 08/2002, http://www . uni-regensburg.de/Fakultaeten/ nat_Fak_I/RAAG.

[Dr1] L. VAN DEN DrIEs, Tarski's problem and Pfaffian functions, in: "Logic colloquium '84" (Manchester, 1984), Stud. Logic Found. Math. 120, North-Holland, Amsterdam, 1986, pp. 59-90.

[Dr2] L. VAN DEN DRIES, "Tame topology and o-minimal structures", London Mathematical Society Lecture Note Series 248, Cambridge University Press, Cambridge, 1998.

[DrM] L. VAN DEN Dries ET C. Miller, Geometric categories and o-minimal structures, Duke Math. J. 84(2) (1996), 497-540.

[Fe] H. Federer, "Geometric measure theory", Die Grundlehren der mathematischen Wissenschaften 153, Springer-Verlag New York Inc., New York, 1969.

[Ga] A. M. GabriÈlov, Projections of semianalytic sets, (Russian), Funkcional. Anal. i Priložen 2(4) (1968), 18-30.

[Go] C. Godbillon, "Géométrie différentielle et mécanique analytique", Hermann, Paris, 1969.

[KM] M. Karpinski et A. Macintyre, A generalization of Wilkie's theorem of the complement, and an application to Pfaffian closure, Selecta Math. (N.S.) 5(4) (1999), 507-516.

[Kh] A. G. KhovanskiI, Real analytic manifolds with the property of finiteness, and complex abelian integrals, (Russian), Funktsional. Anal. i Prilozhen. 18(2) (1984), 40-50.

[K1] K. KURDYKA, Une généralisation de l'inégalite de gradient pour les applications analytiques, conférence donnée au Séminaire de Géométrie Algébrique Réelle de Rennes le 6 décembre 2001.

[K2] K. Kurdyka, Travail en cours de rédaction. 
[LR] J.-M. LiOn ET J.-P. Rolin, Volumes, feuilles de Rolle de feuilletages analytiques et théorème de Wilkie, Ann. Fac. Sci. Toulouse Math. (6) 7(1) (1998), 93-112.

[LS] J.-M. Lion et P. Speissegger, Analytic stratification in the Pfaffian closure of an o-minimal structure, Duke Math. J. 103(2) (2000), 215-231.

[Ło] S. ŁoJASIEWICZ, Une propriété topologique des sous-ensembles analytiques réels, in: "Les équations aux dérivées partielles" (Paris, 1962), Éditions du Centre National de la Recherche Scientifique, Paris, 1963, pp. 87-89.

[MR] R. Moussu et C. A. Roche, Théorèmes de finitude pour les variétés pfaffiennes, Ann. Inst. Fourier (Grenoble) 42(1-2) (1992), 393-420.

[Sp] P. Speissegger, The Pfaffian closure of an o-minimal structure, J. Reine Angew. Math. 508 (1999), 189-211.

[Ta] A. TARsKI, "A decision method for elementary algebra and geometry", 2nd ed., University of California Press, Berkeley and Los Angeles, Calif., 1951.

[Wi] A. J. Wilkie, A theorem of the complement and some new o-minimal structures, Selecta Math. (N.S.) 5(4) (1999), 397-421.

F. Chazal:

Université de Bourgogne

Institut de Mathématiques de Bourgogne

UMR $5584 \mathrm{du}$ C.N.R.S.

UFR des Sciences et Techniques

9 avenue Alain Savary, B.P. 47870

21078 Dijon Cedex

France

E-mail address: fchazal@u-bourgogne.fr

J.-M. Lion:

IRMAR

Université de Rennes 1-CNRS

Campus de Beaulieu

35042 Rennes Cedex

France

E-mail address: jean-marie.lion@univ-rennes1.fr

Primera versió rebuda el 25 de setembre de 2002, darrera versió rebuda el 10 de març de 2003. 